\title{
Culture-independent assessment of planktonic ciliate diversity in coastal northwest Atlantic waters
}

\author{
Mary Doherty ${ }^{1}$, Barbara A. Costas ${ }^{2}$, George B. McManus ${ }^{2}$, Laura A. Katz ${ }^{1,3, *}$ \\ ${ }^{1}$ Graduate Program in Organismic and Evolutionary Biology, University of Massachusetts, 611 North Pleasant Street, \\ Amherst, Massachusetts 01002, USA \\ ${ }^{2}$ Department of Marine Sciences, University of Connecticut, 1080 Shennecosett Road, Groton, Connecticut 06340, USA \\ ${ }^{3}$ Department of Biological Sciences, Clark Science Center, Smith College, Northampton, Massachusetts 01063, USA
}

\begin{abstract}
Planktonic ciliates within the subclasses Choreotrichia and Oligotrichia play critical roles in food webs in the world's oceans. To assess the diversity of these ciliates, we designed primers specific to small subunit ribosomal DNA (SSU rDNA) of ciliates within these clades and sampled at 3 coastal sites in the northwest Atlantic in October 2004 and May 2005. We also preserved and analyzed samples using standard ecological methods to compare observations from light microscopy with our molecular results. We found that (1) estimates of diversity based on molecular markers were similar to estimates from morphological observations for choreotrich ciliates, but much greater for oligotrich ciliates; (2) while similar levels of diversity were found at each site, each collection had its own distinct assemblage of rare and abundant ciliate haplotypes; (3) genealogical analyses of our samples combined with published sequences from identified morphospecies revealed that haplotype diversity at these sites is greatest within the genus Strombidium, in the Oligotrichia. The results from this ciliate-specific analysis are consistent with previous molecular studies on microbial diversity in marine systems in that they reveal high diversity and shifting assemblages within microbial communities.
\end{abstract}

KEY WORDS: Ciliate phylogeography $\cdot$ Microzooplankton $\cdot$ Diversity $\cdot$ Oligotrichia $\cdot$ Choreotrichia Strombidium · Culture independent

\section{INTRODUCTION}

Elucidating patterns of ciliate diversity in marine systems is essential because ciliates play key roles in marine food webs. Ciliates are a trophic link between nano- and picoplankton, and larger metazoan organisms (Pierce \& Turner 1992, Calbet \& Saiz 2005), and are important grazers on harmful algal bloom species (Rosetta \& McManus 2003, Kamiyama \& Matsuyama 2005). Microbial assemblages shift and change with biotic and abiotic processes (Fuhrman et al. 2006), and hence identifying the abundance and diversity of key organisms is essential to understanding ecosystem functions.

The present study combines molecular methods and microscopy to elucidate the diversity of 2 ecologically important subclasses of ciliates: Oligotrichia and Choreotrichia. These 2 morphologically diverse subclasses dominate ciliate communities in near-coastal waters. For example, in a 3 yr study in Long Island Sound, Capriulo \& Carpenter (1983) and Capriulo et al. (2002) reported the presence of 65 species from these groups, based on light microscopy (30 species of tintinnids, 12 other choreotrich species, and 23 oligotrichs).

The use of morphological characteristics alone to assess ciliate community diversity is problematic in ecological studies, since for many ciliates identification to species level is possible only using fine structure. For example, some ciliate groups abundant in the plankton can only be distinguished to coarse morphological categories, such as 'small aloricate ciliates' (Sherr et al. 1986, Fileman \& Leakey 2005), or as highly 
diverse assemblages within a genus, such as Strombidium spp. (Modigh 2001, Fileman \& Leakey 2005). One exception is the tintinnids, a group of choreotrichs with a rich literature of morphological species descriptions based mainly on size and shape of the lorica (outer sheath). Ocean transect studies of tintinnid diversity reveal distinct assemblages within ocean provinces, with specific diversity patterns characterizing each area (Modigh et al. 2003, Thompson 2004). Further, compiling 451 data points from the literature for tintinnid diversity, Dolan et al. (2006) found a latitudinal distribution of tintinnid richness.

Contemporary views on diversity of marine microbes have been altered by large-scale, molecular surveys (Caron et al. 2004, DeLong 2005, Giovannoni \& Stingl 2005, Richards \& Bass 2005, Xu 2006). Such studies revealed considerable microbial diversity not captured by culture-dependent methods. With these new methods, it is possible to sample from a broader range of microbial habitats (Dawson \& Pace 2002, Edgcomb et al. 2002, Zettler et al. 2002, Lopez-Garcia et al. 2003), to sample previously undetected microbes (Diez et al. 2001, Moon-van der Staay et al. 2001), and to determine the spatial and temporal scales of microbial diversity (Romari \& Vaulot 2004, Behnke et al. 2006, Cordova-Kreylos et al. 2006, Ley et al. 2006). Although ciliates are often captured in studies of eukaryotic diversity, we know of no published reports focusing specifically on ciliate diversity using cultureindependent molecular methods.

We combined molecular methods and microscopy to identify ciliates in the subclasses Oligotrichia and Choreotrichia across 2 seasons (fall and spring) at 3 geographically distinct coastal sites in the northwest Atlantic. In addition to evaluating morphological and genetic diversity, we measured a few key environmental parameters that may influence ciliate distributions.

\section{MATERIALS AND METHODS}

Collection. Ciliates were sampled from 3 near-shore locations: Bucks Harbor (BH) and Southport Island (SI) in the Gulf of Maine, and Groton, Connecticut (CT), in Long Island Sound (Fig. 1, Table 1). Samples were collected on the same day for 2 time points, one in October 2004 and one in May 2005. For molecular analysis, we collected 50 to 601 of surface water per sample and concentrated the microplankton down to 11 by siphoning through a $20 \mu \mathrm{m}$ mesh. Of this $1 \mathrm{l}$, five $200 \mathrm{ml}$ aliquots were filtered onto a $5 \mu \mathrm{m}$ Millipore cellulose nitrate filter. Each filter was placed in $1 \mathrm{ml}$ of DNA prep buffer (100 mM NaCl, Tris-EDTA at $\mathrm{pH}$ 8, and $0.5 \%$ SDS) until DNA extraction. For microscopic examination, $250 \mathrm{ml}$ surface water samples were preserved in $5 \%$ Lugol's iodine solution.

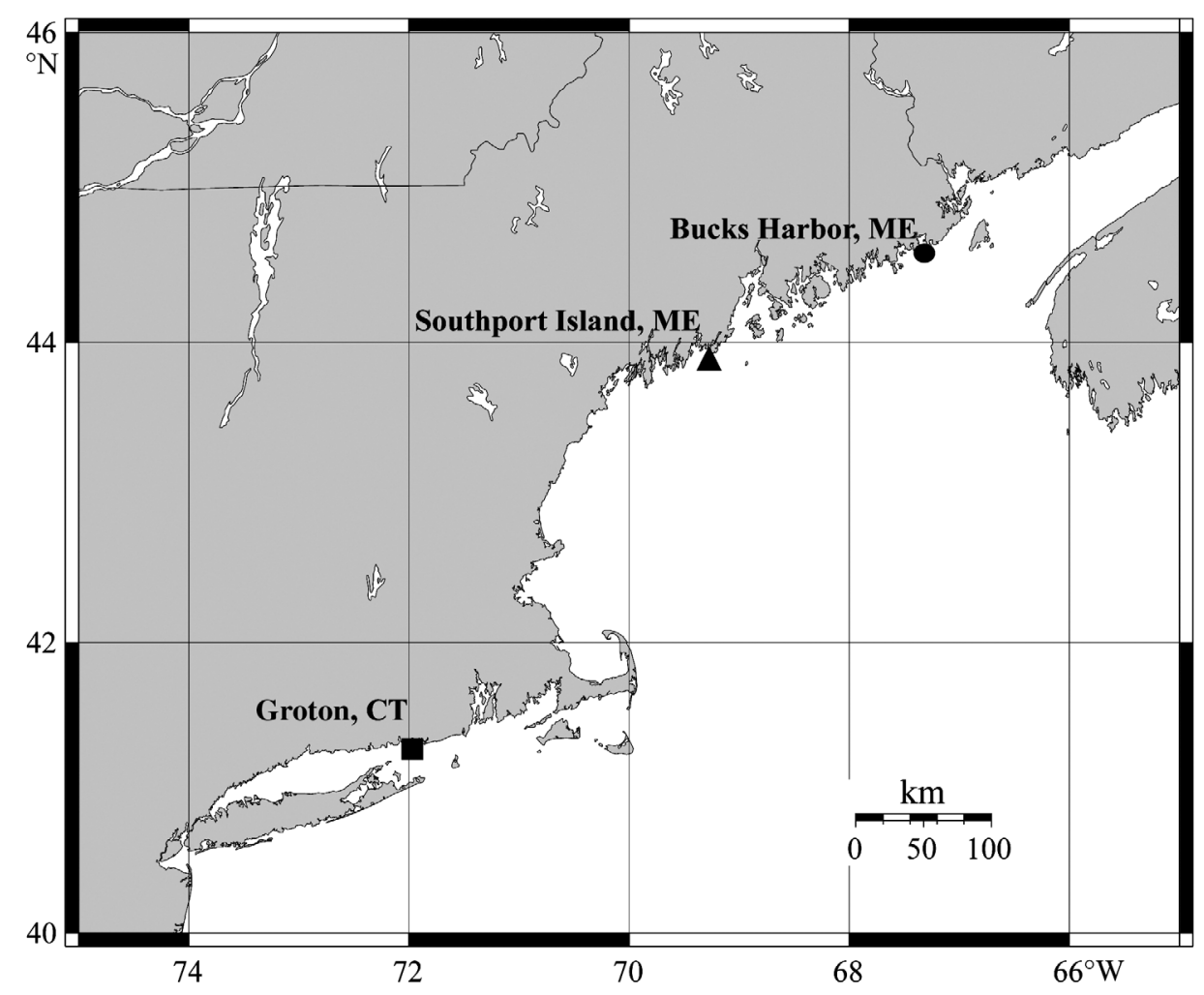

Fig. 1. Location of the 3 collection sites. Symbols representing each site on the map are used in Figs. 2, 4, \& 5 to identify location 
Table 1. Sampling data for the 3 collection sites and 2 time points, showing temperature, salinity, and chlorophyll concentration. NA: not available

\begin{tabular}{|lclccc|}
\hline Location & Abbreviation & Sampling date & Temp. $\left({ }^{\circ} \mathrm{C}\right)$ & Salinity (ppt) & Chlorophyll $\left(\mu \mathrm{g} \mathrm{l}^{-1}\right)$ \\
\hline Bucks Harbor, ME & BH & Oct 2004 & 11.1 & 33 & NA \\
$\left(44^{\circ} 38.20^{\prime} \mathrm{N}, 67^{\circ} 22.29^{\prime} \mathrm{W}\right)$ & & May 2005 & 6.7 & 30 & 2.0 \\
Southport Island, ME & SI & Oct 2004 & 13.3 & 32 & NA \\
$\left(43^{\circ} 49.05^{\prime}\right.$ N, 69 $\left.39.16^{\prime} \mathrm{W}\right)$ & & May 2005 & 8.9 & 27 & 10.4 \\
Groton, CT & CT & Oct 2004 & 18.8 & 29 & NA \\
$\left(41^{\circ} 19.00^{\prime} \mathrm{N}, 72^{\circ} 03.65^{\prime} \mathrm{W}\right)$ & & May 2005 & 12.1 & 25 & 1.4 \\
\hline
\end{tabular}

We recorded ambient temperature and salinity on each collection day. For the May 2005 samples, we also measured chlorophyll concentration. For chlorophyll, $100 \mathrm{ml}$ of each pre-concentrated water sample was filtered onto a Whatman GF/F glass fiber filter. The filter was folded in half, wrapped in aluminum foil and placed on dry ice, and later stored at $-80^{\circ} \mathrm{C}$ prior to extraction in $90 \%$ aqueous acetone and quantification by fluorescence.

DNA extraction, primer design, and amplification. Total genomic DNA was extracted from the samples using standard protocols (Ausubel et al. 1992). Two of the samples (Southport Island, October [SI Oct]; Connecticut, May [CT May]) were difficult to amplify, so we extracted these filters with the DNeasy plant kit by Qiagen (cat. \# 69104) to remove compounds inhibitory to PCR.

To design clade-specific primers, we searched GenBank for all full-length small subunit ribosomal DNA (SSU rDNA) sequences from the ciliate class Spirotrichea. We aligned a total of 69 sequences representing all 5 subclasses (Oligotrichia, Choreotrichia, Hypotrichia, Stichotrichia, and Protocruziidia) using the ClustalW algorithm in the program MegAlign (DNAStar). We then determined optimal sites for primer design by visual inspection in MacClade version 4.06. Our aim was to choose primers in regions that are conserved within the Choreotrichia and Oligotrichia, but divergent in the other subclasses. However, there were very few regions in the Spirotrichea that were specific to only Oligotrichia and Choreotrichia without also matching the Stichotrichia, so we allowed for some overlap in sequence with this clade. Our rationale was that Stichotrichs are predominantly sediment dwelling and unlikely to dominate planktonic samples. The 4 primers differed from the nearest sister taxa (members of the Hypotrichia) by an average of $22.4 \%$ (4.75 bases) with a range of 15 to $33 \%$ (3 to 7 bases). To increase specificity, we designed the primers to concentrate the divergence at the $3^{\prime}$ ends. We selected primer pairs from 2 distinct, nonoverlapping regions of the SSU marker to compare efficiency in capturing ciliate community diversity using published sequences from identified morphospecies as the backbone for our analyses. The efficiency of these primer pairs was tested by amplifying genomic DNA of cultured specimens within the Choreotrichia and Oligotrichia, including Metacylis angulata, Laboea strobila, Strombidium oculatum, Strombidium stylifer, Tintinnopsis sp., and Eutintinnus pectinis.

We amplified DNA fragments using polymerase chain reaction (PCR) with Phusion polymerase and reagents from Finnzyme. Each sample was amplified using both primer sets. The first primer pair (primer set A) consisted of 150+: 5' AHTTACATGGATAACCGTGG and 568-: 5' GGTSTAAATTCRKYTCATTKC. Cycling conditions for primer set A were as follows: $98^{\circ} \mathrm{C}$ for $2 \mathrm{~min}$, 35 cycles of $98^{\circ} \mathrm{C}$ for $15 \mathrm{~s}, 55^{\circ} \mathrm{C}$ for $30 \mathrm{~s}$, and $72^{\circ} \mathrm{C}$ for $45 \mathrm{~s}$, then a $10 \mathrm{~min}$ extension at $72^{\circ} \mathrm{C}$. The second primer pair (primer set B) consisted of 1199+: 5' GCCGACTCGGGATCGGGGGC and 1765-: 5' CCCCAKCACGACDCMTATTGCTG. Cycling conditions for primer set B were as follows: $98^{\circ} \mathrm{C}$ for $2 \mathrm{~min}, 35$ cycles of $98^{\circ} \mathrm{C}$ for $15 \mathrm{~s}, 72^{\circ} \mathrm{C}$ for $1.5 \mathrm{~min}$, then a $10 \mathrm{~min}$ extension at $72^{\circ} \mathrm{C}$. Three separate $20 \mu$ reactions were run and the products pooled to minimize PCR bias. Primer set A amplifies a $418 \mathrm{bp}$ fragment of the SSU rDNA gene, and primer set B amplifies a 566 bp region.

PCR products were gel-isolated, and cleaned using the UltraClean GelSpin DNA purification kit from Mo Bio Laboratories (cat. \#12400-100). We used the pSTBlue-1 Perfectly Blunt Cloning Kit from Novagen (cat. \# 70191-4DFRZ) for cloning, and then picked and miniprepped 192 colonies per sample using either the Plasmid 96 Miniprep Kit from Edge Biosystems (cat. \# 49181) or the PureLink 96 Plasmid Purification System from Invitrogen (cat. \# 12263-018). Sequencing reactions were performed using Big Dye Termination Kit (Applied Biosystems), cleaned with a sephadex plate column, and sequenced on an ABI 377 automated sequencer.

Genealogical analyses. We assembled and edited sequences using SeqMan (DNAStar). We explored assembling our sequences at different similarity cutoffs in SeqMan, which allows assembly of haplotypes with varying parameters (e.g. allowing sequences to 
cluster that are within $1 \%$ of one another). Based on comparison of similarities set from 90 to $100 \%$, we selected a $99 \%$ similarity cutoff for genealogical analyses and diversity estimation to allow for inclusion of highly related but distinct taxa. Haplotypes were then checked for identity with published sequences using BLAST search (Altschul et al. 1997) on NCBI. For genealogical analyses, haplotypes were aligned with published sequences from identified morphospecies obtained by searching GenBank for all entries recorded as Choreotrichia and Oligotrichia. Although included in our BLAST assessments, we chose to exclude environmental samples from our genealogical analyses in order to focus on a taxonomic framework for interpreting our haplotypes. We used the CLUSTAL $\mathrm{W}$ algorithm as implemented in MegAlign to align our sequences with the published sequences. We finalized alignments by eye in MacClade version 4.06.

To check our assembled haplotype sequences for PCR artifacts such as chimeras, we scanned our alignments in both the Chimaera (Maynard Smith 1992, Posada \& Crandall 2001, Posada 2002) and GENECONV (Padidam et al. 1999) applications in Recombination Detection Program (RDP) version 2.0, recombination detection software (Martin et al. 2005). We then visually inspected the aligned sequences in MacClade version 4.06 to detect recombination events. We detected recent recombination, or PCR chimerism, in only one haplotype, sequenced by primer set $\mathrm{A}$, and removed it from the analysis.

Bayesian analyses were conducted for each primer dataset using a GTR + G + I model of sequence evolution in MrBayes (Ronquist \& Huelsenbeck 2003). Four simultaneous MCMCMC chains were run for 3000000 generations, sampling every 100 generations. Stationarity in likelihood scores was determined by plotting the $-\operatorname{lnL}$ against the generation. All trees below the observed stationarity level were discarded, resulting in a 'burn in' of 75000 generations. Estimation of best fit models for partial SSU rDNA gene sequences were performed using MrModeltest 2.2 (Nylander 2004 MrModeltest v2. Evolutionary Biology Centre, Uppsala University; program distributed by the author).

Morphological identification. We estimated the number of morphospecies in the sample by settling 2 aliquots of $100 \mathrm{ml}$ each and examining the entire settled volume at 400 to $600 \times$ on an Olympus inverted microscope. Morphospecies were documented by drawing and digital photography. Using this approach, most ciliates were recognizable to genus based on overall morphology. For the tintinnids, an extensive literature on species diagnosis is available, based on lorica morphol- ogy. Unfortunately, the lorica has been shown to be a plastic characteristic within species (Alder 1999). For non-tintinnid choreotrichs, and for oligotrichs, species diagnosis is based on silver-staining procedures and/or electron microscopy, methods that are best applied when an abundance of specimens is available, for example from cultures (Montagnes \& Lynn 1991, Lynn \& Small 2000). Moreover, the fixative that provides the best quantitative preservation, Lugol's iodine solution, obscures many cytological features. For these reasons, we used simple light microscopy and examined our samples conservatively, identifying separate morphospecies only when clear differences in morphology were present. Principally, we used the keys in Marshall (1969) for tintinnids and in Maeda \& Carey (1985) and Maeda (1986) for other choreotrichs and for oligotrichs.

Diversity estimation. To compare species diversity between molecular samples, rarefaction curves were calculated using EstimateS version 7.5 (Colwell 2005), comparing number of clones sequenced to number of observed haplotypes based on our 99\% assembling criterion. We also calculated the non-parametric richness estimator (Chao1) with EstimateS using 100 randomizations, sampling without replacement.

\section{RESULTS}

Our clone libraries generated a rich diversity of haplotypes within the Choreotrichia and Oligotrichia. The clade-specific primers were successful in amplifying the target groups of Oligotrichia and Choreotrichia, and excluding non-target groups, as evidenced in our phylogenies (Fig. 2). The few exceptions to this were in the sister taxa, Stichotrichia (Fig. 2b). In spite of the degeneracy in the primer sequences, our phylogenies and BLAST search results showed no evidence of amplification of non-ciliates, or even of nonSpirotrichs (Fig. 2).

We sequenced a total of 731 clones with primer set A, and 653 clones with primer set B (Table 2). To explore the patterns of divergence among the resulting sequences, we assessed the number of haplotypes generated using a range of similarity cutoffs at 15 intervals,

Table 2. Diversity of haplotypes sampled, including the percentage of common (sequenced $\geq 50$ times in the 6 samples) and rare (sequenced $\leq 2$ times in the 6 samples) haplotypes

\begin{tabular}{|lccccc|}
\hline $\begin{array}{l}\text { Primer } \\
\text { set }\end{array}$ & Region & $\begin{array}{c}\text { Clones } \\
\text { sampled }\end{array}$ & $\begin{array}{c}\text { No. of distinct } \\
\text { haplotypes }\end{array}$ & $\begin{array}{c}\text { \% common } \\
\text { haplotypes }\end{array}$ & $\begin{array}{c}\text { \% rare } \\
\text { haplotypes }\end{array}$ \\
\hline A & $150+, 568-$ & 731 & 56 & 7.0 & 54 \\
B & $1199+, 1765-$ & 653 & 66 & 1.4 & 78 \\
\hline
\end{tabular}


a

Primer Set A

BH Oct

BH May

$\triangle$ SI Oct

$\triangle$ SI May

$\square$ CT Oct

$\square$ CT May

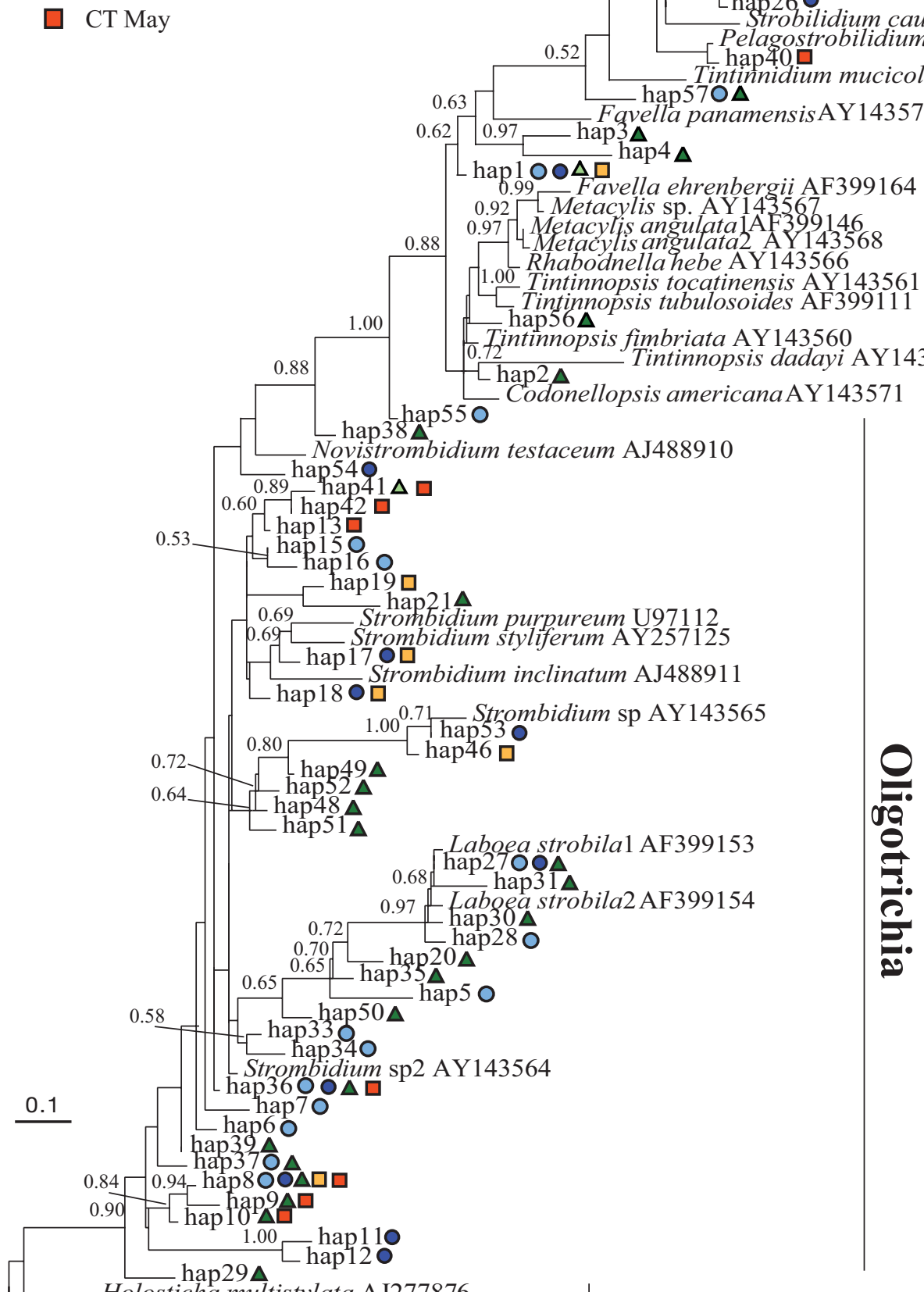

Fig. 2. (This and following page.) Bayesian phylogenies based on the analysis of partial SSU rDNA gene sequences. Topologies shown are trees with the highest likelihood scores. Numbers at nodes are Bayesian posterior probabilities. All branches are drawn to scale. (a) Phylogeny (likelihood score -4546.744) of 56 environmental haplotypes (hap) sequenced with primer set A with 34 published sequences, based on 375 characters. (b) Phylogeny (likelihood score -4559.868) of 67 environmental haplotypes (hbp) sequenced with primer set B with 34 published sequences, based on 477 characters. Symbols next to haplotype names indicate where and at which time point each haplotype was found (BH: Bucks Harbor, Maine; SI: Southport Island, Maine; CT: Groton, Connecticut-see Fig. 1)

Holosticha multistylata AJ277876 Holosticha diademata DO059583 1.00 Halteria grandinella2 AY 0074 Gonostomum strenuum AJ310493 Protocruzia adherens AY217727

\section{Outgroups}


b

\section{Primer Set B}

BH Oct

○ BH May

$\triangle$ SI Oct

$\triangle$ SI May

$\square$ CT Oct

$\square$ CT May

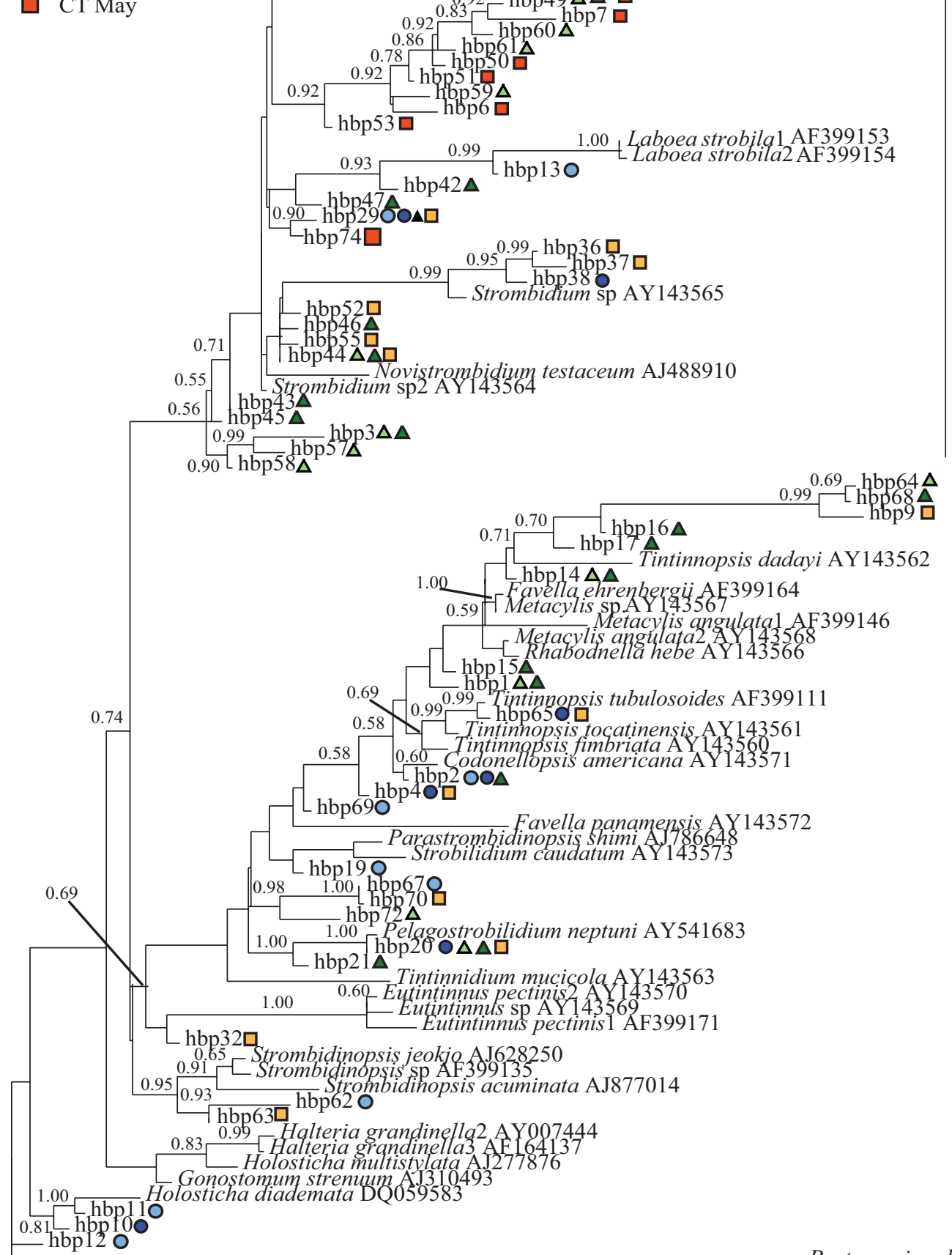

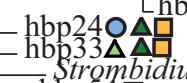


collapsing sequences that were 90 to $100 \%$ identical into single haplotypes (see 'Materials and methods'). Analysis of the effect of this range of bins with the 2 primer sets revealed a large increase in the number of haplotypes between 97 and 99\% similarity (Fig. 3), with minimal change when increased to $100 \%$. In other words, there are few clones that differ from one another by $\leq 1 \%$, and this level of diversity includes experimental error. Thus, for the purposes of comparing sites and generating genealogies, we used the $99 \%$ assembly criterion.

Based on an assembly of clones that were $\geq 99 \%$ similar, 56 and 66 haplotypes were generated from primer sets A and B, respectively (Table 2) (GenBank Accession Numbers EF553335-EF553457). Of these haplotypes, we found only one (hbp23, amplified with primer set B) that occurred at all sites and times, and a small proportion (13 of 123) of the haplotypes were sampled in 3 or more of the 6 collections (see Appendix 1). The bulk of the haplotypes we sequenced with both primer sets (110 of 123) were rare, occurring in only 1 or 2 samples. This rarity was consistent across both primer sets, representing $89 \%$ of the haplotypes in both cases (see Appendix 1).

Four of the 56 haplotypes obtained with primer set A were $100 \%$ identical to published sequences as determined by BLAST (Altschul et al. 1997): hap40 = Pelagostrobilidium neptuni (GB AY541683), hap44 = Strombidinopsis jeokjo (AJ628250), hap27 = Laboea strobila (AF399153), and hap36 = Strombidium biarmatum (AY541684) (Table 3). Four of the 66 haplotypes sequenced with primer set B were $100 \%$ identical to published sequences: hbp65 = Tintinnopsis tubulosoides (AF399111), hbp71 = an uncultured eukaryote (AY129053), hbp20 = Strombidium biarmatum (AY541684) and hbp23 = Pelagostrobilidium neptuni (AY541683) (Table 3). The latter two were captured with both primer sets A and B.

We sampled the greatest diversity within the Oligotrichia (Fig. 2). Primer sets A and B sequenced 38 of 56

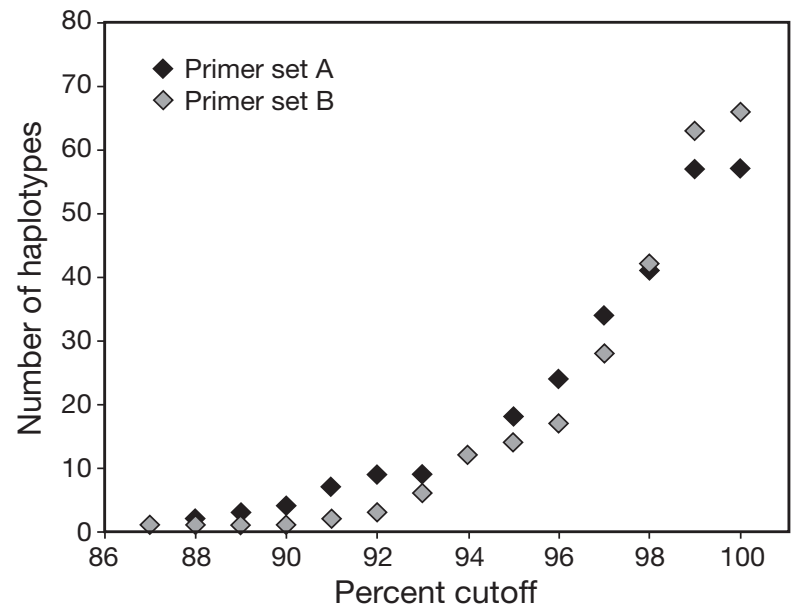

Fig. 3. Total diversity of ciliate SSU rDNA haplotypes sampled with primer sets $\mathrm{A}$ and $\mathrm{B}$ using different percent similarity cutoffs. We use a $99 \%$ cutoff for genealogies and diversity estimates

and 42 of 66 haplotypes that fell within this clade, respectively. Our most common and abundant haplotype was identical to published sequences of Strombidium biarmatum. This haplotype sequence was sampled 184 times with primer set A and 251 times with primer set B (Table 3) and falls within the Oligotrichia clade as hap36 (Fig. 2a) and hbp23 (Fig. 2b), respectively. Within the Choreotrichia, the 2 primer sets captured similar levels of diversity. Primer set A sampled 19 of 56 haplotypes within this clade, and primer set B sampled 21 of 66 haplotypes. We detected non-target sequences from the closely related group, Stichotrichia, in 3 of our samples using primer set $\mathrm{B}$. These included a haplotype $98 \%$ similar to Holosticha diademata (DQ059583) in the BH Oct sample, and a haplotype $96 \%$ similar to Gonostomum strenuum (AJ310493) in the BH May and CT Oct samples (Fig 2b).

Two of the samples we collected (SI Oct and CT May) were difficult to amplify with PCR, and required

Table 3. Haplotypes sampled with $100 \%$ identity to published sequences, including number of samples containing the haplotype, and number of clones sequenced with the haplotype

\begin{tabular}{|c|c|c|c|c|}
\hline Taxon hit with BLAST search & $\begin{array}{c}\text { Genbank } \\
\text { Accession No. }\end{array}$ & $\begin{array}{l}\text { Haplotype } \\
\text { No. }\end{array}$ & $\begin{array}{l}\text { No. of collections } \\
\text { containing haplotype }\end{array}$ & $\begin{array}{l}\text { Clones sequenced } \\
\text { with haplotype }\end{array}$ \\
\hline \multicolumn{5}{|l|}{ Primer set A } \\
\hline Laboea strobila & AF399153 & hap27 & 3 & 65 \\
\hline Strombidium biarmatum & AY541684 & hap36 & 4 & 184 \\
\hline Strombidinopsis jeokjo & AJ628250 & hap44 & 3 & 17 \\
\hline Pelagostrobilidium neptuni & AY541683 & hap40 & 1 & 4 \\
\hline \multicolumn{5}{|l|}{ Primer set B } \\
\hline Pelagostrobilidium neptuni & AY541683 & hbp20 & 4 & 23 \\
\hline Strombidium biarmatum & AY541684 & hbp23 & 6 & 251 \\
\hline Tintinnopsis tubulosoides & AF399111 & hbp65 & 2 & 8 \\
\hline Uncultured eukaryote & AY129053 & hbp71 & 1 & 11 \\
\hline
\end{tabular}


a plant-specific extraction protocol to obtain genomic DNA suitable for PCR (see 'Materials and methods'). The 2 samples were much lower in haplotype diversity than any of the others when amplified using primer set A, but not with primer set B (Table 4). In addition, morphological and molecular results are inconsistent in the CT May sample, which was quantified using both methods (Table 5). Choreotrichs that were present in the sample did not amplify, while numbers of oligotrichs similar to the other samples did amplify. These data suggest the water sampled at the CT May and the SI Oct sites contained a component inhibitory to PCR and/or that the community composition in these samples contain fewer members, as seen in another study (Costas et al. in press).

We calculated rarefaction curves, again based on haplotypes defined to be $99 \%$ similar (Fig. 5), using EstimateS version 7.5 (Colwell 2005). Rarefaction calculates the number of haplotypes as a function of the number of sequences sampled, and is a means of comparing diversity across different sample sizes. We estimated diversity within each sample collected and plotted the curves for each on the same graph to compare haplotype richness between samples (Fig. 5). The trajectories of the curves indicate that we have not sampled all of the diversity for the collection sites, as the slopes of the lines have not reached an asymptote (Fig. 5). Comparing diversity between collections, the $95 \%$ confidence intervals overlap, indicating no significant differences in diversity as

Table 4 . Chao1 estimates of sample diversity (95\% confidence intervals in parentheses) for each primer set. See Table 1 for abbreviations

\begin{tabular}{|lcc|}
\hline Sample & Primer set A & Primer set B \\
\hline BH Oct & $171(90.9-223.8)$ & $171(90.9-223.8)$ \\
BH May & $91(50.8-196.1)$ & $78(43.9-130.8)$ \\
SI Oct & $6(2.6-20.7)$ & $136(73.8-191.3)$ \\
SI May & $325(161.0-347.7)$ & $253(129.1-292.8)$ \\
CT Oct & $105(58.0-160.3)$ & $153(82.1-207.4)$ \\
CT May & $28(15.8-64.4)$ & $78(43.9-130.8)$ \\
\hline
\end{tabular}

Table 5. Ciliate morphospecies identified by light microscopy (M) from May 2005, along with the number of haplotypes sequenced $(H)$ in these clades from the same sample. Highest number across primer sets is reported for haplotype number. CT haplotype numbers show evidence of difficulty in amplification by PCR. See Table 1 for abbreviations

\begin{tabular}{|lccccccc|}
\hline \multirow{2}{*}{ Taxon } & \multicolumn{2}{c}{ BH } & \multicolumn{2}{c}{ SI } & \multicolumn{2}{c|}{ CT } \\
& M & H & M & H & M & H \\
\hline Choreotrichs & & & & & & \\
Tintinnids (Tintinnopsis spp., Eutintinnus sp.) & 3 & 3 & 6 & 7 & 4 & 0 \\
Strobilidium, Strombidinopsis, Lohmaniella spp. & 3 & 3 & 4 & 3 & $4-5$ & 0 \\
Oligotrichs & & & & & & \\
Strombidium spp. & 5 & 9 & 4 & 17 & $4-5$ & 12 \\
Laboea strobila & 1 & 1 & 1 & 1 & 1 & 0 \\
\hline
\end{tabular}

estimated by rarefaction. There is a trend toward higher diversity in the SI May sample based on the rarefaction trajectories for both primer sets A and B (Fig. 5). The SI Oct and CT May samples sequenced with primer set A have lower diversity, having a shallower slope than the other curves. These were the samples that were difficult to amplify.

We used the Chao1 diversity estimator to estimate total haplotype diversity of the samples (Table 4). This is a non-parametric estimator of the total diversity in the community from which the sample is drawn. Using 100 randomizations without replacement, we estimated diversity against number of clones sampled for each collection separately for the 2 primer sets, and reported the total estimated diversity (Table 4 ). The data from the Chao1 estimator is consistent with our rarefaction data. SI May had the highest estimates of total diversity (325 and 253 haplotypes for primers A and B, respectively; Table 4). Our results for the SI Oct and CT May samples were inconsistent across primer sets $A$ and $B$, reflected in the Chao1 diversity estimates. Based on results from primer set $A_{\text {, the Chao1 }}$ estimate of total diversity was only 6 haplotypes for SI Oct, and 28 for CT May (Table 4). Using the data from primer set B for each of these samples, SI Oct was estimated to contain 136 haplotypes and CT May was estimated to contain 78 haplotypes (Table 4). Estimated sample diversity was not correlated with the abiotic factors we measured (temperature, salinity, latitude and season; Table 1). However, there was a trend of higher haplotype diversity estimated by rarefaction and Chao1 (Fig. 5, Table 4) associated with high chlorophyll content in the SI May sample.

As is typical for spring in Long Island Sound, ciliates from the CT May sample were abundant in the plankton

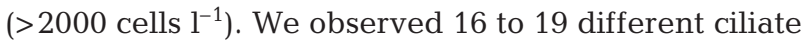
morphospecies in $200 \mathrm{ml}$ total of settled sample. The majority of these were from the spirotrich subclasses Oligotrichia and Choreotrichia (the latter including the tintinnids; Table 5). The only ciliate that was identified to the species level was Laboea strobila, easily recognizable by its spirally wound girdle and large size (McManus \& Fuhrman 1986). This mixotrophic oligotrich is common in Long Island Sound during spring and early summer. Our molecular approach did not capture $L$. strobila at this sampling location and time (Fig. 2). There were at least 4 other distinct morphospecies within the same family (Strombidiidae), including probably Strombidium conicum (Table 5).

Microscopic observations suggested that the spring ciliate assemblage in 

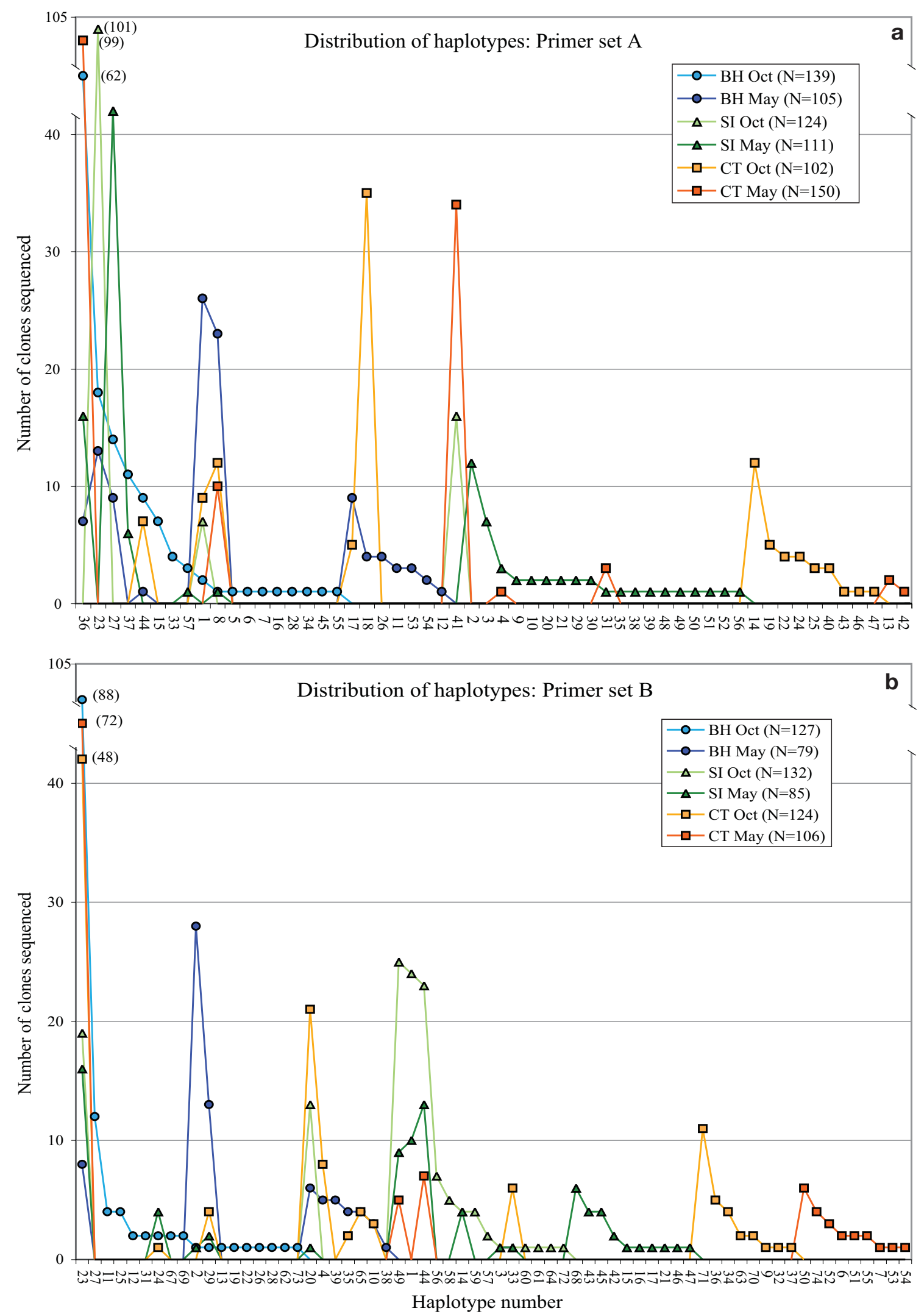

Fig. 4. Distribution of rare and abundant ciliate SSU rDNA haplotypes across sampling locations and times for (a) primer set A and (b) primer set B. Each sample collected has a distinct assemblage of rare and abundant haplotypes. See Fig. 2 for abbreviations 

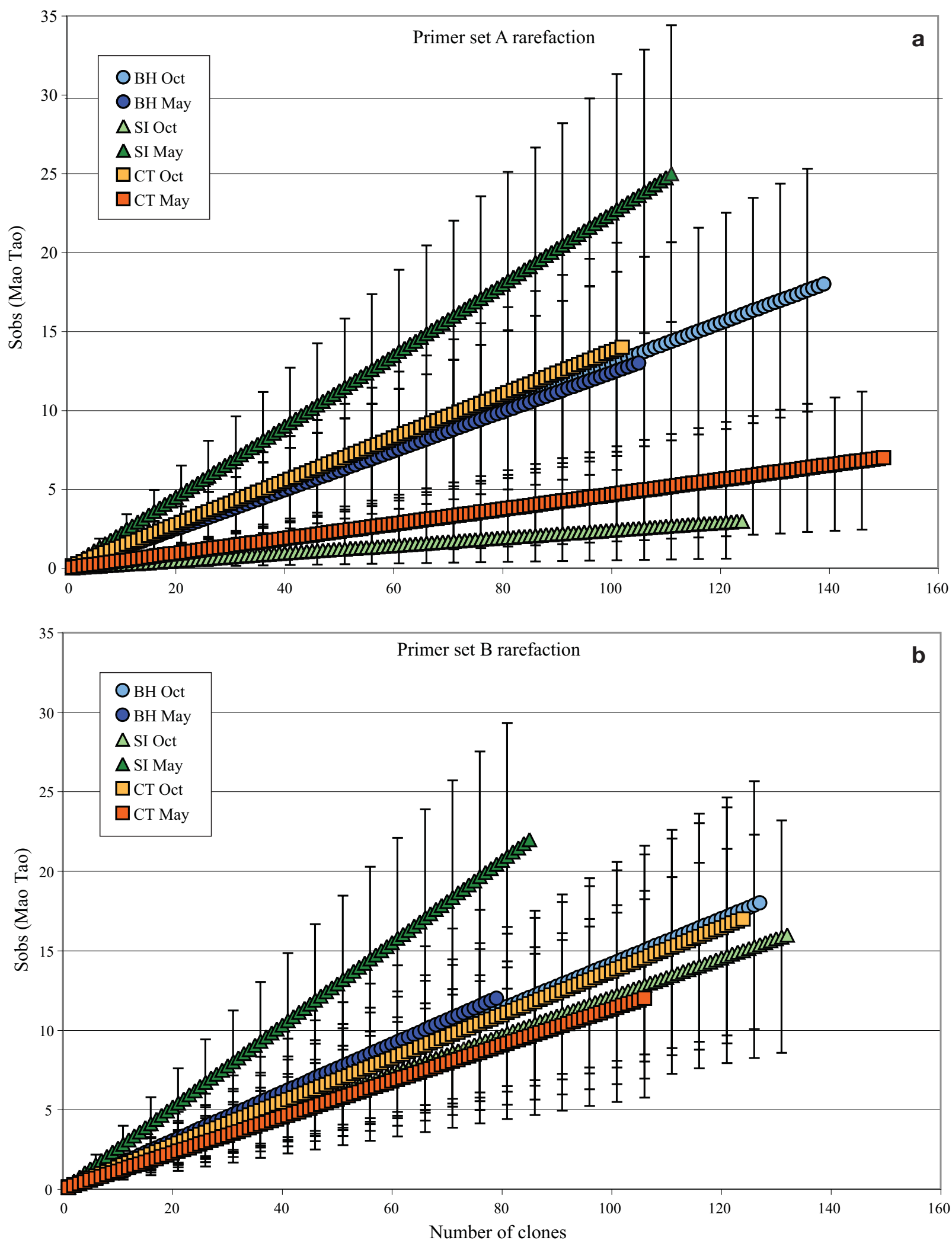

Fig. 5. Rarefaction curves calculated with EstimateS version 7.5 for (a) primer set A and (b) primer set B. Estimated diversity (Sobs, Mao Tao) versus number of clones sampled for each collection is shown with $95 \%$ confidence intervals. The sample collected in May at Southport Island (SI May) has the steepest trajectory for both primers, indicating higher ciliate diversity within the sample. See Fig. 2 for abbreviations 
the plankton of coastal Maine was similar to that sampled simultaneously from Long Island Sound. Laboea strobila was present in all 3 samples, with higher abundances at SI (ca. 300 cells $\mathrm{l}^{-1}$ ). Molecular samples are consistent with these observations at both Maine locations. SI clone libraries also contain L. strobila in high abundances (Fig. 2, Appendix 1). Other strombidiids could be classified into 4 to 5 morphospecies. Most of the tintinnids at both sites were from the agglutinated genus Tintinnopsis. One empty lorica of the hyaline genus Parafavella (probably $P$. denticulata) was observed in the SI sample (Table 5). Our molecular genealogies show that in Maine we sampled haplotypes closely related to published Tintinnopsis and Parfavella genera (Fig. 2).

\section{DISCUSSION}

We observed similar overall levels of diversity across 3 sites and 2 seasons, with only a few haplotypes found multiple times in clone libraries. The limitations inherent in using PCR of environmental samples to assess abundance and diversity are well known and we recognize that, in the absence of comparisons with quantitative PCR results, assessments of frequencies in clone libraries are at best a rough approximation of abundance in nature. The potentially differing levels of amplification in ciliate macronuclear genomes further exacerbate this problem. We attempted to reduce the bias in our amplification methods by running multiple PCR reactions and pooling the product pairs in generating clone libraries. In addition, we tested the cloning results with one of our samples by sequencing twice the number of clones, and found no difference in the diversity estimates with additional sampling. Moreover, we found that our clade-specific primers were consistent in the levels of diversity they identified, and that our methods were reproducible: when we repeated the PCR, cloning and sequencing methods for 2 of the samples, we saw no major differences between trials.

Although the level of diversity was similar across sites (with the possible exception of the elevated diversity at the chlorophyll-rich SI Oct site), there is a unique assemblage of haplotypes at each sampling location and time (Fig. 4). This contrasts with some studies based on morphological observations of overall ciliate diversity, which reveal a striking homogeneity at local and global scales of ciliate species assemblages (Finlay 2002). Our findings are consistent with the studies on tintinnids (Modigh et al. 2003, Thompson 2004, Dolan et al. 2006), where specific patterns of diversity are associated with regional areas in the ocean. The observation in our study is that morphospecies underestimate genetic diversity. Nevertheless, similarity between our molecular results and the tintinnid data indicate that a pattern of varying assemblages may prevail within planktonic ciliates.

The estimates of overall diversity vary between the molecular and morphological sampling methods. Based on microscopic observation using standard ecological techniques, we conservatively estimated that 12 to 15 morphospecies from the Choreotrichia and Oligotrichia were present in each sample. Based on our molecular data, Chao1 diversity calculated with EstimateS version 7.5 (Colwell 2005) for each set of haplotypes sampled is substantially higher (Table 4). The average estimated diversity of haplotypes per collection is 133, an approximately 100-fold difference from the morphospecies estimate. Possible explanations for the apparent underestimate of morphological methods include (1) cryptic species of ciliates, (2) high intraspecific variation within morphospecies, and (3) undercounting due to lower volume sampled for the time-intensive morphological estimates. Distinguishing among these hypotheses must await further molecular and genetic analyses of morphospecies.

When we look within the subclasses, we find that diversity estimates based on morphological observations are consistent with haplotype frequencies in the Choreotrichia, but not in the Oligotrichia. For BH and SI in Maine, we observed approximately the same number of tintinnid and other choreotrich morphospecies as haplotypes sequenced (Table 5). The CT May sample, which was difficult to amplify, is not consistent with this trend, but we predict that this is a result of PCR inhibition in that specific sample (Table 5). In contrast with the Choreotrichia, the number of haplotypes sequenced in the Oligotrichia, particularly those falling within the Strombidium genus on our phylogeny (Fig. 2), was 2 to 3 times greater than the number of distinct morphospecies we observed microscopically from this clade (Table 5). The greater diversity of genetic versus morphological entities within our samples suggests that there may be cryptic species of ciliates that are not readily distinguishable by light microscopy in the Oligotrichia, or that there is an as yet unappreciated diversity of very small ciliates, which are difficult to quantify by microscopy (Sherr et al. 1986).

To assess the taxonomic position of haplotypes, we generated genealogies including published sequences from identified morphospecies and excluded unknown environmental samples (Fig. 2). Notably, the bulk of the diversity we observed is within the Strombidium spp. clade, which yielded 37 (primer set A) and 42 (primer set B) haplotypes (Fig. 2). Given that a total of 63 morphospecies have been described in this clade worldwide (Agatha 2004), we would not have predicted such high numbers of haplotypes falling within this clade purely from marine planktonic samples. The most abundant and widespread haplotype we sampled 
was $100 \%$ identical to Strombidium biarmatum, found in high numbers and in almost every sample (Appendix 1). If the genetic diversity we obtained is reflective of species diversity, the number of haplotypes observed in the Strombidium clade may indicate high levels of ecological complexity within marine microenvironments, where many similar species are able to coexist by fine-scale partitioning of niches. Alternatively, these data may reflect high levels of standing genetic variation within a small number of ciliate species. Data on other planktonic ciliates within these subclasses are not consistent with this second assertion (Katz et al. 2005), as we find extremely low levels of genetic variation $(<0.5 \%)$ in Laboea strobila populations sampled over space and time.

Although the relationships between genetic and morphological species of ciliates are unclear, some have argued that a $1 \%$ cutoff provides information about species diversity for eukaryotic microbes for fulllength SSU rDNA sequences (Richards et al. 2005, Stoeck et al. 2006). Using this $99 \%$ similarity cutoff between sequences as a criterion for defining a haplotype, we find many more genetic entities than morphological entities in comparable samples. Notably, for Choreotrichia and Oligotrichia, the levels of genetic variation in the 2 regions assessed here were comparable to divergences for the full-length sequence. To assess the relevance of a $1 \%$ cutoff, we tabulated distance between data from species and genera. Pairwise genetic distances between SSU rDNA sequences from published morphospecies within Choreotrichia and Oligotrichia vary widely, ranging from 0 to $3.7 \%$ divergence between species, while distances between genera range from 1.7 to $8.7 \%$ divergence.

Comparisons of diversity within Oligotrichia by assembling at a lower percentage of similarity indicate that the number of haplotypes does not drop to the levels estimated by morphology until we get to $<95 \%$ similarity. Prior studies combining morphological and molecular data also indicate high genetic variation within morphologically similar members of the Oligotrichia (Snoeyenbos-West et al. 2002, Katz et al. 2005).

Haplotype diversity in our samples is consistent with environmental samples of marine eukaryotes in general (Countway et al. 2005). Universal eukaryotic primers detected high numbers of rare haplotypes, and relatively few abundant haplotypes, and were able to detect changing community compositions within a sample. Recent analyses of prokaryotic diversity in the coastal Pacific Ocean revealed predictable patterns of change in community composition over seasonal and interannual scales (Fuhrman et al. 2006). Thus, the genetic variation we see in ciliate assemblages may indicate adaptations to diverse and variable microbial communities at lower trophic levels.
By sampling with primers designed to amplify 2 different regions of the SSU rDNA locus, we identified differences within a single sample that may be the result of preferential amplification of DNA templates. However, our phylogenetic data and our diversity estimates were comparable across primer sets, containing similar numbers of haplotypes within the higher taxonomic groupings of Choreotrichia and Oligotrichia (Fig. 2). Ultimately, this preliminary work can be applied to additional studies designing FISH probes, or amplification of samples using the forward primer of set A and the reverse primer of set $\mathrm{B}$ to obtain a larger sequence.

Using clade-specific primers and focusing on these 2 groups of ciliates has allowed us to sample these coastal environments more thoroughly than previous environmental studies. Our data reveal complex diversity patterns across time and space. In addition, we find that molecular tools enabled us to sample diversity not found using morphological approaches. In the Oligotrichia, we observed no greater than 6 morphospecies in total, compared to the 38 to 40 haplotypes we sampled with primer sets A and B (Table 5). We can use these to look for patterns and driving forces of ciliate diversity, and for morphological attributes that correlate with the molecular diversity.

Acknowledgements. This work was supported by the National Science Foundation (OCE-0221137) to L.A.K. and G.B.M.

\section{LITERATURE CITED}

Agatha S (2004) A cladistic approach for the classification of oligotrichid ciliates (Ciliophora: Spirotrichia). Acta Protozool 43:201-217

Alder V (1999) Tintinnoinea. In: Boltovskoy D (ed) South Atlantic zooplankton. Backhuys, Leiden, p 321-384

Altschul SF, Madden TL, Schäffer AA, Zhang J, Zhang Z, Miller W, Lipman DJ (1997) Gapped BLAST and PSI-BLAST: a new generation of protein database search programs. Nucleic Acids Res 25:3389-3402

Ausubel FM, Brent R, Kingston RE, Moore DD, Seidman JG, Smith JA, Struhl K (1992) Current protocols in molecular biology. Wiley-Liss, New York

Behnke A, Bunge J, Barger K, Breiner HW, Alla V, Stoeck T (2006) Microeukaryote community patterns along an $\mathrm{O}_{2} / \mathrm{H}_{2} \mathrm{~S}$ gradient in a supersulfidic anoxic Fjord (Framvaren, Norway). Appl Environ Microbiol 72:3626-3636

Calbet A, Saiz E (2005) The ciliate-copepod link in marine ecosystems. Aquat Microb Ecol 38:157-167

Capriulo GM, Carpenter EJ (1983) Abundance, species composition and feeding impact of tintinnid micro-zooplankton in central Long Island Sound. Mar Ecol Prog Ser 10:277-288

Capriulo GM, Smith G, Troy R, Wikfors GH, Pellet J, Yarish C (2002) The planktonic food web structure of a temperate zone estuary, and its alteration due to eutrophication. Hydrobiologia 475:263-333

Caron DA, Countway PD, Brown MV (2004) The growing contributions of molecular biology and immunology to protistan ecology: molecular signatures as ecological tools. J Eukaryot Microbiol 51:38-48 
Colwell RK (2005) EstimateS: statistical estimation of species richness and shared species from samples. Version 7.5. User's guide and application. Available at http://purl.oclc.org/estimates

Cordova-Kreylos AL, Cao YP, Green PG, Hwang HM and 5 others (2006) Diversity, composition, and geographical distribution of microbial communities in California salt marsh sediments. Appl Environ Microbiol 72:3357-3366

Costas B, McManus G, Doherty M, Katz L (in press) Use of speciesspecific primers and PCR to measure the distributions of planktonic ciliates in coastal waters. Limnol Oceanogr Methods

Countway PD, Gast RJ, Savai P, Caron DA (2005) Protistan diversity estimates based on 18S rDNA from seawater incubations in the western North Atlantic. J Eukaryot Microbiol 52:95-106

Dawson SC, Pace NR (2002) Novel kingdom-level eukaryotic diversity in anoxic environments. Proc Natl Acad Sci USA 99:8324-8329

DeLong EE (2005) Microbial community genomics in the ocean. Nat Rev Microbiol 3:459-469

Díez B, Pedrós-Alió C, Massana R (2001) Study of genetic diversity of eukaryotic picoplankton in different oceanic regions by small-subunit rRNA gene cloning and sequencing. Appl Environ Microbiol 67:2932-2941

Dolan JR, Lemee R, Gasparini S, Mousseau L, Heyndrickx C (2006) Probing diversity in the plankton: using patterns in Tintinnids (planktonic marine ciliates) to identify mechanisms. Hydrobiologia 555:143-157

Edgcomb VP, Kysela DT, Teske A, Gomez AD, Sogin ML (2002) Benthic eukaryotic diversity in the Guaymas Basin hydrothermal vent environment. Proc Natl Acad Sci USA 99:7658-7662

Fileman ES, Leakey RJG (2005) Microzooplankton dynamics during the development of the spring bloom in the north-east Atlantic. J Mar Biol Assoc UK 85:741-753

Finlay BJ (2002) Global dispersal of free-living microbial eukaryote species. Science 296:1061-1063

Fuhrman JA, Hewson I, Schwalbach MS, Steele JA, Brown MV, Naeem S (2006) Annually reoccurring bacterial communities are predictable from ocean conditions. Proc Natl Acad Sci USA 103:13104-13109

Giovannoni SJ, Stingl U (2005) Molecular diversity and ecology of microbial plankton. Nature 437:343-348

Kamiyama T, Matsuyama Y (2005) Temporal changes in the ciliate assemblage and consecutive estimates of their grazing effect during the course of a Heterocapsa circularisquama bloom. J Plankton Res 27: 303-311; doi:10.1093/plankt/fbi006

Katz LA, McManus GB, Snoeyenbos-West OLO, Griffin A, Pirog K, Costas B, Foissner W (2005) Reframing the 'Everything is everywhere' debate: evidence for high gene flow and diversity in ciliate morphospecies. Aquat Microb Ecol 41:55-65

Ley RE, Harris JK, Wilcox J, Spear JR and 6 others (2006) Unexpected diversity and complexity of the Guerrero Negro hypersaline microbial mat. Appl Environ Microbiol 72: 3685-3695

Lopez-Garcia P, Philippe H, Gail F, Moreira D (2003) Autochthonous eukaryotic diversity in hydrothermal sediment and experimental microcolonizers at the Mid-Atlantic Ridge. Proc Natl Acad Sci USA 100:697-702

Lynn D, Small EB (2000) Phylum Ciliophora DOFLEIN, 1901. In: Lee J, Leedale G, Bradbury P (eds) The illustrated guide to protozoa, Vol 1. Society of Protozoologists, Lawrence, KS, p 371-656

Maeda M (1986) An illustrated guide to the species of the families Halteriidae and Strobilidiidae (Oligotrichida, Ciliophora) free swimming protozoa common in the aquatic environment. Bull Ocean Res Inst Univ Tokyo 21:1-67

Maeda M, Carey PG (1985) An illustrated guide to the species of the family Strombidiidae (Oligotrichida, Ciliophora), free swimming protozoa common in the aquatic environment. Bull Ocean Res Inst Univ Tokyo 19:1-68
Marshall SM (1969) Protozoa-Order Tintinnida. Cons Int Explor Mer, Zooplankton Sheets 117-127:1-81

Martin DP, Williamson C, Posada D (2005) RDP2: recombination detection and analysis from sequence alignments. Bioinformatics 21:260-262

Maynard Smith J (1992) Analysing the mosaic structure of genes. J Mol Evol 34:126-129

McManus GB, Fuhrman JA (1986) Photosynthetic pigments in the ciliate Laboea strobila from Long Island Sound, USA. J Plankton Res 8:317-327

Modigh M (2001) Seasonal variations of photosynthetic ciliates at a Mediterranean coastal site. Aquat Microb Ecol 23:163-175

Modigh M, Castaldo S, Saggiomo M, Santarpia I (2003) Distribution of tintinnid species from 42 degrees $\mathrm{N}$ to 43 degrees $\mathrm{S}$ through the Indian Ocean. Hydrobiologia 503:251-262

Montagnes DJS, Lynn DH (1991) Taxonomy of choreotrichs, the major marine ciliates, with emphasis on the aloricate forms. Mar Microb Food Webs 5:59-74

Moon-van der Staay SY, De Wachter R, Vaulot D (2001) Oceanic $18 \mathrm{~S}$ rDNA sequences from picoplankton reveal unsuspected eukaryotic diversity. Nature 409:607-610

Padidam M, Sawyer S, Fauquet CM (1999) Possible emergence of new geminiviruses by frequent recombination. Virology 265:218-225

Pierce RW, Turner JT (1992) Ecology of planktonic ciliates in marine food webs. Rev Aquat Sci 6:139-181

Posada D (2002) Evaluation of methods for detecting recombination from DNA sequences: Empirical data. Molec Biol Evol 19:708-717

Posada D, Crandall KA (2001) Intraspecific gene genealogies: trees grafting into networks. Trends Ecol Evol 16:37-45

Richards TA, Bass D (2005) Molecular screening of free-living microbial eukaryotes: diversity and distribution using a metaanalysis. Curr Opinion Microbiol 8:240-252

Richards TA, Vepritskiy AA, Gouliamova DE, Nierzwicki-Bauer SA (2005) The molecular diversity of freshwater picoeukaryotes from an oligotrophic lake reveals diverse, distinctive and globally dispersed lineages. Environ Microbiol 7:1413-1425

Romari K, Vaulot D (2004) Composition and temporal variability of picoeukaryote communities at a coastal site of the English Channel from 18S rDNA sequences. Limnol Oceanogr 49: 784-798

Ronquist F, Huelsenbeck JP (2003) MrBayes 3: Bayesian phylogenetic inference under mixed models. Bioinformatics 19: $1572-1574$

Rosetta CH, McManus GB (2003) Feeding by ciliates on two harmful algal bloom species, Prymnesium parvum and Prorocentrum minimum. Harmful Algae 2:109-126

Sherr EB, Sherr BF, Fallon RD, Newell SY (1986) Small, aloricate ciliates as a major component of the marine heterotrophic nanoplankton. Limnol Oceanogr 31:177-183

Snoeyenbos-West OLO, Salcedo T, McManus GB, Katz LA (2002) Insights into the diversity of choreotrich and oligotrich ciliates (class: Spirotrichea) based on genealogical analyses of multiple loci. Int J Syst Evol Microbiol 52:1901-1913

Stoeck T, Hayward B, Taylor GT, Varela R, Epstein SS (2006) A multiple PCR-primer approach to access the microeukaryotic diversity in environmental samples. Protist 157:31-43

Thompson GA (2004) Tintinnid diversity trends in the southwestern Atlantic Ocean (29 to $60^{\circ} \mathrm{S}$ ). Aquat Microb Ecol 35:93-103

$\mathrm{Xu}$ JP (2006) Microbial ecology in the age of genomics and metagenomics: concepts, tools, and recent advances. Molec Ecol 15:1713-1731

Zettler LAA, Gomez F, Zettler E, Keenan BG, Amils R, Sogin ML (2002) Eukaryotic diversity in Spain's River of Fire-this ancient and hostile ecosystem hosts a surprising variety of microbial organisms. Nature 417:137 
Appendix 1. Haplotypes sequenced with primer set A (hap) and primer set B (hbp), indicating number of clones sequenced per haplotype, per sample. BH: Bucks Harbor, Maine; SI: Southport Island, Maine; CT: Groton, Connecticut

\begin{tabular}{|c|c|c|c|c|c|c|c|c|c|c|c|c|c|}
\hline \multirow{2}{*}{$\begin{array}{l}\text { Haplotype } \\
\text { Primer A }\end{array}$} & \multicolumn{2}{|c|}{$\mathrm{BH}$} & \multicolumn{2}{|c|}{ SI } & \multicolumn{2}{|c|}{$\mathrm{CT}$} & \multirow{2}{*}{$\begin{array}{l}\text { Haplotype } \\
\text { Primer B }\end{array}$} & \multicolumn{2}{|c|}{$\mathrm{BH}$} & \multicolumn{2}{|c|}{ SI } & \multicolumn{2}{|c|}{ CT } \\
\hline & Oct & May & Oct & May & Oct & May & & Oct & May & Oct & May & Oct & May \\
\hline hap01 & 2 & 26 & 7 & & 9 & & hbp01 & & & 24 & 10 & & \\
\hline hap02 & & & & 1 & & & hbp02 & 1 & 28 & & 1 & & \\
\hline hap03 & & & & 1 & & & hbp03 & & & 1 & 1 & & \\
\hline hap04 & & & & 1 & & & hbp04 & & 5 & & & 8 & \\
\hline hap05 & 1 & & & & & & hbp06 & & & & & & 2 \\
\hline hap06 & 1 & & & & & & hbp07 & & & & & & 1 \\
\hline hap07 & 1 & & & & & & hbp09 & & & & & 1 & \\
\hline hap08 & 1 & 23 & & 1 & 12 & 10 & hbp10 & & 3 & & & 3 & \\
\hline hap09 & & & & 3 & & 1 & hbp11 & 4 & & & & & \\
\hline hap10 & & & & 1 & & 3 & hbp12 & 2 & & & & & \\
\hline hap11 & & 3 & & & & & hbp13 & 1 & & & & & \\
\hline hap12 & & 1 & & & & & hbp14 & & & 4 & 4 & & \\
\hline hap13 & & & & & & 1 & hbp15 & & & & 1 & & \\
\hline hap14 & & & & & 3 & & hbp16 & & & & 1 & & \\
\hline hap15 & 7 & & & & & & hbp17 & & & & 1 & & \\
\hline hap16 & 1 & & & & & & hbp19 & 1 & & & & & \\
\hline hap17 & & 9 & & & 5 & & hbp20 & & 6 & 13 & 1 & 21 & \\
\hline hap18 & & 4 & & & 35 & & hbp21 & & & & 1 & & \\
\hline hap19 & & & & & 1 & & hbp 22 & 1 & & & & & \\
\hline hap20 & & & & 2 & & & hbp23 & 88 & 8 & 19 & 16 & 48 & 72 \\
\hline hap21 & & & & 2 & & & hbp 24 & 2 & & & 4 & 1 & \\
\hline hap22 & & & & & 1 & & hbp25 & 4 & & & & & \\
\hline hap23 & 18 & 13 & 101 & & & & hbp 26 & 1 & & & & & \\
\hline hap24 & & & & & 5 & & hbp27 & 12 & & & & & \\
\hline hap25 & & & & & 1 & & hbp28 & 1 & & & & & \\
\hline hap26 & & 4 & & & & & hbp29 & 1 & 13 & & 2 & 4 & \\
\hline hap27 & 14 & 9 & & 42 & & & hbp31 & 2 & & & & & \\
\hline hap28 & 1 & & & & & & hbp32 & & & & & 1 & \\
\hline hap29 & & & & 2 & & & hbp33 & & & 1 & 1 & 6 & \\
\hline hap30 & & & & 2 & & & hbp34 & & & & & 4 & \\
\hline hap31 & & & & 1 & & & hbp35 & & 4 & & & 2 & \\
\hline hap33 & 4 & & & & & & hbp36 & & & & & 5 & \\
\hline hap34 & 1 & & & & & & hbp37 & & & & & 1 & \\
\hline hap35 & & & & 2 & & & hbp38 & & 1 & & & & \\
\hline hap36 & 62 & 7 & & 16 & & 99 & hbp39 & & 5 & & & & \\
\hline hap37 & 11 & & & 6 & & & hbp42 & & & & 2 & & \\
\hline hap38 & & & & 1 & & & hbp43 & & & & 4 & & \\
\hline hap39 & & & & 1 & & & hbp 44 & & & 23 & 13 & & 7 \\
\hline hap40 & & & & & 4 & & hbp45 & & & & 4 & & \\
\hline hap41 & & & 16 & & & 34 & hbp46 & & & & 1 & & \\
\hline hap42 & & & & & & 2 & hbp47 & & & & 1 & & \\
\hline hap43 & & & & & 4 & & hbp49 & & & 25 & 9 & & 5 \\
\hline hap44 & 9 & 1 & & & 7 & & hbp50 & & & & & & 6 \\
\hline hap45 & 1 & & & & & & hbp51 & & & & & & 2 \\
\hline hap46 & & & & & 12 & & hbp52 & & & & & & 3 \\
\hline hap47 & & & & & 3 & & hbp53 & & & & & & 1 \\
\hline hap48 & & & & 12 & & & hbp54 & & & & & & 1 \\
\hline hap49 & & & & 2 & & & hbp55 & & & & & & 2 \\
\hline hap50 & & & & 1 & & & hbp56 & & & 7 & & & \\
\hline hap51 & & & & 1 & & & hbp57 & & & 2 & & & \\
\hline hap52 & & & & 1 & & & hbp58 & & & 5 & & & \\
\hline hap53 & & 3 & & & & & hbp59 & & & 4 & & & \\
\hline hap54 & & 2 & & & & & hbp60 & & & 1 & & & \\
\hline hap55 & 1 & & & & & & hbp61 & & & 1 & & & \\
\hline hap56 & & & & 7 & & & hbp62 & 1 & & & & & \\
\hline hap57 & 3 & & & 1 & & & hbp63 & & & & & 2 & \\
\hline & & & & & & & hbp64 & & & 1 & & & \\
\hline & & & & & & & hbp65 & & 4 & & & 4 & \\
\hline & & & & & & & hbp67 & 2 & & & & & \\
\hline & & & & & & & hbp68 & & & & 6 & & \\
\hline & & & & & & & hbp69 & 2 & & & & & \\
\hline & & & & & & & hbp70 & & & & & 2 & \\
\hline & & & & & & & hbp71 & & & & & 11 & \\
\hline & & & & & & & hbp72 & & & 1 & & & \\
\hline & & & & & & & hbp73 & 1 & & & & & \\
\hline & & & & & & & hbp74 & & & & & & 4 \\
\hline
\end{tabular}

